\title{
Pain Management After Total Knee Arthroplasty
}

\author{
Hossein Pirmohamadi ${ }^{1}$, Sara Rahmati Roodsari ${ }^{2}$, Zahra Arab-Mazar (iD) ${ }^{3}$ and Mohsen Rahimi ${ }^{4,}$ \\ ${ }^{1}$ Trauma Research Center, Baqiyatollah University of Medical Sciences, Tehran, Iran \\ ${ }^{2}$ Functional Neurosurgery Research Center, Shohada Tajrish Comprehensive Neurosurgical Center of Excellence, Shahid Beheshti University of Medical Sciences, Tehran, Iran \\ ${ }^{3}$ Department of Medical Parasitology and Mycology, School of Public Health, Tehran University of Medical Sciences, Tehran, Iran \\ ${ }^{4}$ Research Center for Prevention of Oral and Dental Diseases, Baqiyatollah University of Medical Sciences, Tehran, Iran \\ "Corresponding author: Research Center for Prevention of Oral and Dental Diseases, Baqiyatollah University of Medical Sciences, Tehran, Iran. Email: \\ mohsen1rahimi@yahoo.com
}

Received 2021 November 14; Revised 2022 January 01; Accepted 2022 January 22.

Keywords: Knee Joint Pain, Multimodal Analgesia, Postoperative Pain

\section{Dear Editor,}

Pain occurs during local TKR secondary to temperature change, trauma-triggered nerve damage, and local inflammation. Local TKR is often associated with pain following tissue damage and the release of inflammatory mediators, such as histamine, substance P, prostaglandins, and bradykinin (Figure 1) (1).

Several methods have been suggested for managing acute pain after TKR, including peripheral nerve blockers, multimodal or epidural analgesia, parental (IV) analgesia (eg, patient-controlled analgesia), and local infiltration analgesia (LIA) (1).

To avoid postoperative pain, it is proposed to administer analgesics or use other pain-relieving methods before introducing surgical incisions. The most effective strategy seems to be a combination of different methods or medications with different action mechanisms to prevent and alleviate pain and avoid multiple drugs (2). This is important, as the recovery of patients after TKA surgery may delay in the case of the inappropriate use of analgesics (3).

A variety of medications and methods have been proposed to alleviate severe pain after surgery, including painrelieving agents, cyclooxygenase-2 inhibitors, opioids, local infiltration, patient-controlled, multimodal, or epidural anesthesia, and peripheral nerve occlusion (4).

The sufficient use of analgesics after surgery can alleviate pain and obviate the need for opioid use, thus reducing the hospitalization period, drug-related complications, and patients' costs. In addition, patients' satisfaction and rehabilitation will improve. To upgrade patient outcomes, surgeons should be aware of the current anesthesia techniques and analgesia regimens used after TKA. Based on a recent meta-analysis, the femoral and sciatic nerves blockade seems to provide the best patient outcomes (5).
Therefore, it is advisable to utilize preoperative combinations of analgesics and postoperative sciatic and femoral nerve block.

\section{Footnotes}

Authors' Contribution: The authors contributed equally to this study.

Conflict of Interests: The authors declare that there is no conflict of interest.

Data Reproducibility: All data generated or analyzed during this study are included in this published article.

Ethical Approval: Not applicable.

Funding/Support: The authors received no specific funding for this work.

\section{References}

1. Vaishya R, Majeed A. Pain management in total knee replacement. Apollo Med.2012;9(4):323-35. doi:10.1016/j.apme.2012.08.012.

2. Gaffney CJ, Pelt CE, Gililland JM, Peters CL. Perioperative Pain Management in Hip and Knee Arthroplasty. Orthop Clin North Am. 2017;48(4):407-19. doi: 10.1016/j.ocl.2017.05.001. [PubMed: 28870302].

3. Baratta JL, Gandhi K, Viscusi ER. Perioperative pain management for total knee arthroplasty. J Surg Orthop Adv. 2014;23(1):22-36. doi: 10.3113/jsoa.2014.0022. [PubMed: 24641894].

4. Li JW, Ma YS, Xiao LK. Postoperative Pain Management in Total Knee Arthroplasty. Orthop Surg. 2019;11(5):755-61. doi: 10.1111/os.12535. [PubMed: 31663286]. [PubMed Central: PMC6819170].

5. Zorrilla-Vaca A, Li J. The role of sciatic nerve block to complement femoral nerve block in total knee arthroplasty: a meta-analysis of randomized controlled trials. J Anesth. 2018;32(3):341-50. doi: 10.1007/s00540-018-2480-1. [PubMed: 29520522]. 

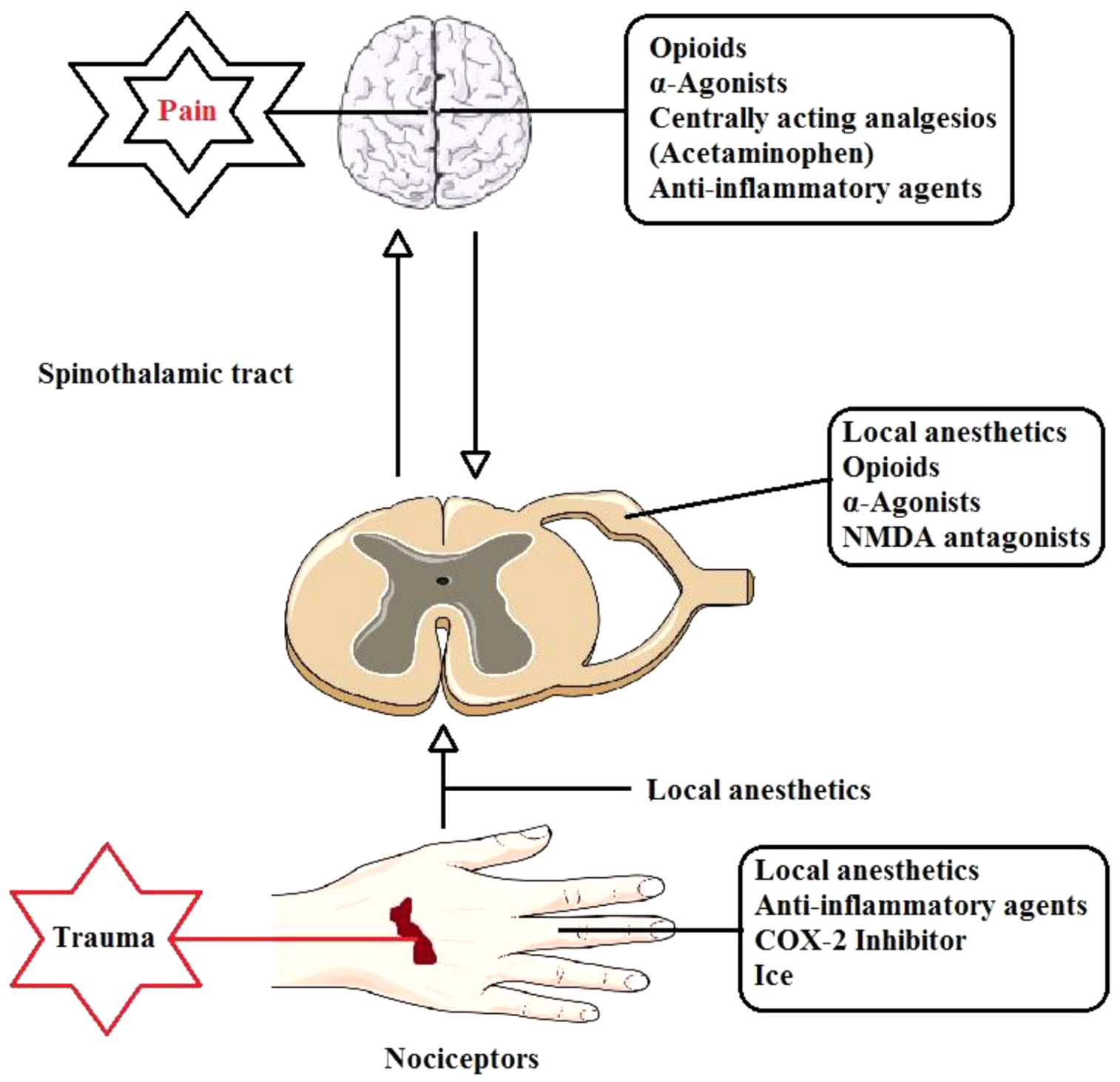

Figure 1. Pain pathways and multimodal analgesic therapy 